\title{
Two Patients with Complex Rearrangements Suggestive of Germline Chromoanagenesis
}

\author{
Priyanka Arya Jennelle C. Hodge Peggy A. Matlock Gail H. Vance \\ Amy M. Breman
}

IU Genetic Testing Laboratories, Department of Medical and Molecular Genetics, Indiana University School of Medicine, Indianapolis, IN, USA

\section{Established Facts}

- Rare constitutional chromoanagenesis events have been reported in a limited number of patients with variable phenotypes.

- A preferential bias for paternal origin has been described in germline chromothripsis-mediated events, but no parental bias has so far been described for chromoanasynthesis-derived rearrangements.

\section{Novel Insights}

- We add 2 additional cases suggestive of constitutional chromoanagenesis to the literature, with our first case representing the oldest known patient.

- Our cases may add evidence to support a paternal bias in constitutional chromoanasynthesis events, similar to what has been observed in cases of germline chromothripsis.

\section{Keywords}

Germline chromoanagenesis - Chromoanasynthesis . Chromothripsis · Complex chromosomal rearrangement . Constitutional events

\section{Abstract \\ Chromoanagenesis, a phenomenon characterized by com- plex chromosomal rearrangement and reorganization events localized to a limited number of genomic regions, in- cludes the subcategories chromothripsis, chromoanasyn-}

thesis, and chromoplexy. Although definitions of these terms are evolving, constitutional chromoanagenesis events have been reported in a limited number of patients with variable phenotypes. We report on 2 cases with complex genomic events characterized by multiple copy number gains and losses confined to a single chromosome region, which are suggestive of constitutional chromoanagenesis. Case 1 is a 43-year-old male with intellectual disability and recently developed generalized tonic-clonic seizures. Chromosomal microarray analysis identified a complex rearrangement involving chromosome region 14q31.1q32.2, consisting of 16
Amy M.Breman

Department of Medical and Molecular Genetics Indiana University School of Medicine 975 W. Walnut St., IB-130 Indianapolis, IN 46202 (USA) abreman@iu.edu 
breakpoints ranging in size from 0.2 to $6.2 \mathrm{Mb}$, with 5 segments of normal copy number present between these alterations. Interestingly, this case represents the oldest known patient with a complex rearrangement indicative of constitutional chromoanagenesis. Case 2 is a 2-year-old female with developmental delay, speech delay, low muscle tone, and seizures. Chromosomal microarray analysis identified a complex rearrangement consisting of 28 breakpoints localized to $18 \mathrm{q} 21.32 \mathrm{q} 23$. The size of the copy number alterations ranged from 0.042 to $5.1 \mathrm{Mb}$, flanked by 12 small segments of normal copy number. These cases add to a growing body of literature demonstrating complex chromosomal rearrangements as a disease mechanism for congenital anomalies.

(c) 2021 S. Karger AG, Basel

\section{Introduction}

Complex chromosomal rearrangements leading to localized structural reorganization of genomic DNA are catastrophic cellular events represented collectively by the term "chromoanagenesis" [Kloosterman et al., 2011; Liu et al., 2011; Stephens et al., 2011; Holland and Cleveland, 2012; Masset et al., 2016]. Analysis of breakpoint junction sequences and investigation of the underlying mechanisms associated with chromoanagenesis has led to the recognition of 3 main categories: chromothripsis, chromoplexy, and chromoanasynthesis [Liu et al., 2011; Stephens et al., 2011; Holland and Cleveland, 2012; Baca et al., 2013; Fukami et al., 2017; Ly and Cleveland, 2017; Pellestor, 2019; Zepeda-Mendoza and Morton, 2019]. Chromothripsis refers to a phenomenon of localized chromosomal shattering and random reorganization of chromosome segments, typically restricted to a single chromosome, and is often associated with deletions [Stephens et al., 2011]. In chromoplexy, derivative chromosomes are generated by a series of chained rearrangements involving segments of DNA from multiple chromosomes that maintain a largely balanced DNA content [Baca et al., 2013]. In contrast, chromoanasynthesis is characterized by alternating copy number changes including a combination of deletions, duplications, and triplications arising from defective replication, typically clustered on a single or a few chromosomes [Liu et al., 2011].

In contrast to chromothripsis and chromoplexy, which have been documented in both cancer and rare constitutional cases, chromoanasynthesis has been primarily associated with complex rearrangements in the germline. Collectively, germline chromoanagenesis-compatible structural rearrangements have been reported in patients with variable neurodevelopmental phenotypes and apparently normal individuals ascertained through an affected family member [Piccione et al., 2010; Kloosterman et al., 2011, 2012; Liu et al., 2011; Genesio et al., 2013, 2015; Gu et al., 2013; Kloosterman and Cuppen, 2013; Fontana et al., 2014; Nazaryan et al., 2014; Plaisancié et al., 2014; de Pagter et al., 2015; Gamba et al., 2015; Wang et al., 2015; Weckselblatt et al., 2015; Anderson et al., 2016; Bertelsen et al., 2016; Burnside et al., 2016; Del Rey et al., 2016; Masset et al., 2016; Fukami et al., 2017; Sabatini et al., 2018; Gudipati et al., 2019; Koltsova et al., 2019; Nazaryan-Petersen et al., 2019; Zepeda-Mendoza and Morton, 2019; Ader et al., 2020]. An additional 2 cases have been identified prenatally [Macera et al., 2015; Bone et al., 2019]. Published cases include inherited chromoanagenesis events, either balanced or unbalanced, as well as those that have arisen de novo during gametogenesis or early embryogenesis. Studies that have identified the parental origin of these events suggest a bias toward paternal alleles in chromothripsis-mediated rearrangements, while no parental bias has been established for chromoanasynthesis events [Kloosterman et al., 2011, 2012; Liu et al., 2011; Weckselblatt and Rudd, 2015; Weckselblatt et al., 2015; Collins et al., 2017; Fukami et al., 2017; Zepeda-Mendoza and Morton, 2019]. Given the overall rarity of chromoanagenesis in the germline, the now-routine clinical use of high-resolution chromosomal microarray analysis (CMA) presents an increased opportunity to uncover these complex events that would have gone largely undetected by conventional cytogenetics, and allows for further exploration of their mechanism and significance in congenital disease.

In this study, we describe localized complex rearrangements in 2 patients evaluated by CMA in our clinical diagnostic laboratory. The genomic rearrangements, each confined to a single chromosome arm, produced an oscillating copy number pattern with segments of disomy present between most of the gains and losses, resembling the characteristic features of germline chromoanagenesis. Additionally, we determined the parental origin of the rearranged chromosomes.

\section{Materials and Methods}

Chromosome Analysis

Peripheral blood specimens were cultured, harvested, and prepared for GTG-banding (G-banding using trypsin and Giemsa) using standard cytogenetic methods. G-banded metaphase chromosomes were analyzed and captured at or above the 500-band level. 


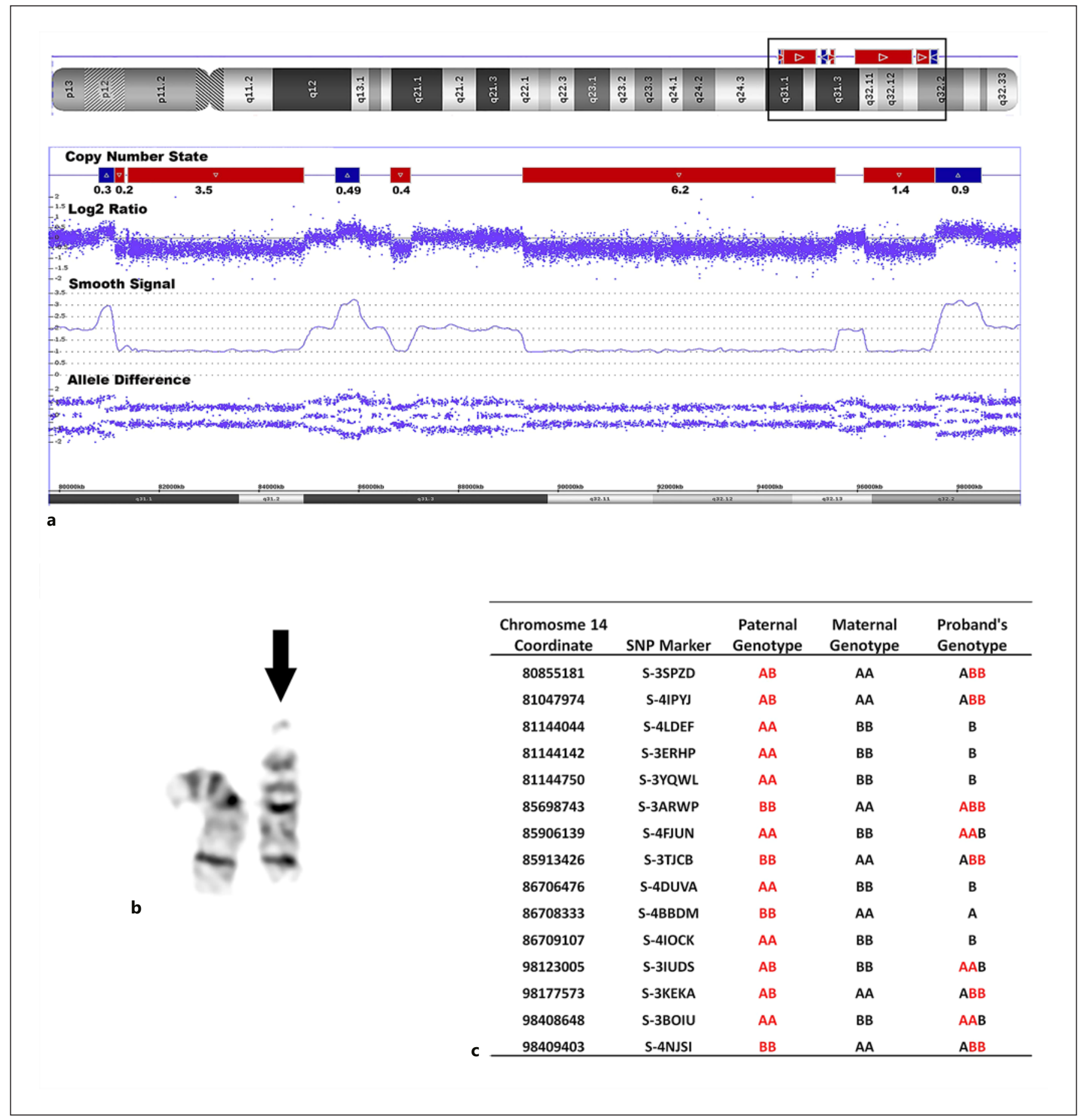

Fig. 1. Complex chromosomal rearrangement involving chromosome 14 in case 1 . a The boxed area on the chromosome 14 idiogram indicates the region of detail shown below it. Chromosomal microarray data depict the complex chromosomal rearrangement confined to $14 q 31.1 q 32.2(80,785,657-98,490,569$ [hg19]). The alternating copy number losses and gains are indicated by the red and blue bars, respectively, with the size of individual CNVs listed below each in Mb. Copy number data (Log2 ratio, smooth signal) and genotype information (allele difference) are represented as in- dividual tracks on the array plot. b G-banded partial karyotype showing the interstitial deletion in one chromosome 14 (arrow). c Genotyping data for the trio are represented by selected informative SNPs with specified chromosomal position and corresponding SNP marker. The paternal genotype is shown in red, the maternal genotype in black, and the derived genotype for the proband is depicted for duplications and deletions along the length of chromosome 14 .
Two Patients with Possible Germline Chromoanagenesis
Cytogenet Genome Res 2020;160:671-679 DOI: $10.1159 / 000512898$ 
Table 1. Specific intervals, size of copy number variants, and genes in the $14 \mathrm{q}$ region for case 1

\begin{tabular}{|c|c|c|c|}
\hline Genomic region [hg19] & $\begin{array}{l}\text { Size, } \\
\mathrm{Mb}\end{array}$ & RefSeq genes in the region & $\begin{array}{l}\text { Classifica- } \\
\text { tion }\end{array}$ \\
\hline 14q31.1(80792017_81108735)×3 & 0.32 & DIO2-AS1, CEP128 & VUS \\
\hline $14 \mathrm{q} 31.1\left(81113098 \_81315110\right) \times 1$ & 0.2 & CEP128 & VUS \\
\hline 14q31.1q31.3(81378210_84909793)×1 & 3.5 & $\begin{array}{l}\text { CEP128, TSHR, GTF2A1, SNORA79, LOC101928504, STON2, LOC100506700, SEL1L, } \\
\text { LINC01467 }\end{array}$ & VUS \\
\hline $14 \mathrm{q} 31.3\left(86647802 \_87050689\right) \times 1$ & 0.4 & No genes & $\begin{array}{l}\text { Likely } \\
\text { benign }\end{array}$ \\
\hline 14q31.3q32.13(89297735_95572111)×1 & 6.2 & $\begin{array}{l}\text { TTC8, FOXN3, FOXN3-AS1, FOXN3-AS2, EFCAB11, TDP1, KCNK13, PSMC1, NRDE2, } \\
\text { CALM1, LINC00642, LOC105370619, TTC7B, LOC101928909, LOC105370622, } \\
\text { RPS6KA5, C14orf159, SNORA11B, GPR68, CCDC88C, PPP4R3A, CATSPERB, TC2N, } \\
\text { FBLN5, TRIP11, ATXN3, NDUFB1, CPSF2, SLC24A4, RIN3, LGMN, GOLGA5, } \\
\text { LOC101929002, CHGA, ITPK1, ITPK1-AS1, MOAP1, TMEM251, C14orf142, UBR7, } \\
\text { BTBD7, UNC79, COX8C, PRIMA1, FAM181A-AS1, FAM181A, ASB2, MIR4506, } \\
\text { LINC00521, OTUB2, DDX24, IFI27L1, IFI27, IFI27L2, PPP4R4, SERPINA10, SERPINA6, } \\
\text { SERPINA2, SERPINA1, SERPINA11, SERPINA9, SERPINA12, SERPINA4, SERPINA5, } \\
\text { SERPINA3, SERPINA13P, GSC, DICER1 }\end{array}$ & Pathogenic \\
\hline 14q32.13q32.2(96143024_97560950)×1 & 1.4 & $\begin{array}{l}\text { TCL1B, TCL1A, TUNAR, C14orf132, BDKRB2, BDKRB1, ATG2B, GSKIP, AK7, } \\
\text { LOC730202, PAPOLA, VRK1, LINC00618 }\end{array}$ & VUS \\
\hline 14q32.2(97561323_98492843)×3 & 0.93 & LOC101929241, LOC100129345, LINC01550 & VUS \\
\hline
\end{tabular}

VUS, variant of uncertain significance.

Chromosomal Microarray Analysis

Genomic DNA was extracted from whole blood using the EZ1 Advanced XL automated DNA extraction method (Qiagen, Hilden, Germany) or from buccal swab samples (ORAcollect Dx kit) using the PrepIT L2P DNA extraction kit (DNA Genotek, Ottawa, ON, Canada). CMA was performed on the patients' DNA using the Applied Biosystems CytoScan HD array (ThermoFisher Scientific, Carlsbad, CA) consisting of approximately 1.9 million copy number and 750,000 SNP oligonucleotide probes. Data analysis was performed using Chromosome Analysis Suite (ChAS) version 3.3.0.139, with the following filtering criteria: deletions $\geq 25 \mathrm{~kb}$ (minimum 25 probes) and duplications $\geq 50 \mathrm{~kb}$ (minimum 50 probes). The results were analyzed and reported using the NCBI human genome build 37.1 (GRCh37/hg19).

\section{SNP Genotype Analysis}

SNP genotype calls were obtained from the ChAS software for the patients and their parents. Trio analysis for Case 1 and duo analysis for Case 2 were performed along the length of the copy number alterations on chromosomes 14 and 18, respectively. For regions of deletion, the SNP genotype was hemizygous in the proband and could be directly compared to the parental genotype. For regions of duplication, SNP genotypes were derived by analyzing allele differences corresponding to $\mathrm{AAA}=1.5, \mathrm{AAB}=0.5, \mathrm{BBA}=$ $-0.5, \mathrm{BBB}=-1.5$. Parent of origin for the rearranged chromosome was determined by comparing the informative SNP calls for the patients and their parents in regions of chromosomal deletion and/ or duplication.

\section{Case Reports and Results}

\section{Case 1}

The patient is a 43-year-old Caucasian male with an extensive medical history that includes developmental delay, intellectual disability, microcephaly, progressive muscle weakness, hypotonia, and late-onset seizures. At birth, he had respiratory distress and was reported to have spina bifida occulta. The patient walked at age 4 and was noted to have speech, cognition, and motor delays. He received special education in school until he was 18 years old. The patient is non-verbal but creates signals and signs to communicate with his mother. He can assist with dressing and feeding himself but otherwise depends on his mother's support. At age 18, G-banded chromosome analysis was performed by an outside laboratory which demonstrated a chromosome 14 deletion. At age 40, the patient developed generalized tonic-clonic seizures, and CMA was requested as part of a neurological workup. His seizures have been managed well with the use of Levetiracetam (Keppra). A recent clinical genetics evaluation identified minor malformations including prominent ears with underdeveloped helices and Darwin tubercles, 
convexity of the nose with a hypoplastic malar region, deep-set eyes with periorbital purple discoloration, and tapered, long thin fingers with slight clubbing. The patient was noted to have markedly reduced muscle tone globally. The family history was notable for maternal recurrent miscarriages.

CMA detected a complex chromosomal rearrangement confined to $14 \mathrm{q} 31.1 \mathrm{q} 32.2$ (coordinates $80,785,657$ $98,492,843$ [hg19]) with 16 breakpoints, including 5 losses and 3 gains for a total of approximately 11.8 and 1.82 $\mathrm{Mb}$ of genomic material, respectively. The size of the copy number variants ranged from 0.2 to $6.2 \mathrm{Mb}$ with 5 segments of normal copy number present between these alterations (Fig. 1a). The genomic coordinates and genes in the affected interval are summarized in Table 1. Most of the copy number changes are of uncertain clinical significance; however, a copy loss of $6.2 \mathrm{Mb}$ at $14 \mathrm{q} 31.3 \mathrm{q} 32.13$ was classified as pathogenic (Table 1). This deletion includes 44 OMIM-annotated genes, and similar deletions have been reported in patients with microcephaly, dysmorphic facial features, intellectual disability, and developmental delay [Piccione et al., 2010]. Conventional Gbanding was performed in conjunction with the CMA study, and results suggested a simple deletion on the distal long arm of chromosome 14 at band 14q32.1, with no evidence of a complex rearrangement (Fig. 1b). The patient's parents were both negative for these copy number rearrangements by CMA (data not shown) indicating this was a de novo event; however, a balanced event in the parents cannot be completely excluded since chromosome analysis was not performed. The parental origin of the abnormal chromosome 14 was determined by SNP genotyping as described in the methods section. In the deleted intervals of $14 \mathrm{q} 31.3 \mathrm{q} 32.13$ in the proband, only maternal alleles were observed, indicating loss of paternal alleles and paternal derivation of the abnormal chromosome 14. Further, the informative SNP markers within the duplicated interval of chromosome 14 were consistent with the additional $14 \mathrm{q}$ segments originating from the paternally derived chromosome (Fig. 1c).

\section{Case 2}

The patient is a 2-year-old female with a history of seizures, developmental delay, poor growth, and hypotonia. There is limited pregnancy/birth history available as the child is in the care of a foster family. Following her birth, the patient had neonatal abstinence syndrome and was reportedly positive for 5 different substances. At 16 months of age, she developed seizures documented by an abnormal EEG, characterized with focal slowing, maxi- mal over the right centroparietal-occipital region, and at times with a sharp configuration. Her seizures are currently under control with the use of Levetiracetam (Keppra). A head CT scan showed an open fontanelle and abnormal metopic suture, while her head MRI was normal. Due to the patient's history of developmental delay and seizures, CMA was requested, and the patient was referred for genetic evaluation following these results. The clinical genetics evaluation at 22 months of age documented mild dysmorphic features including an underdeveloped philtrum, thin vermillion border (both appoximately 4 on Likert scale), and bilaterally low-set ears with normal architecture. The patient has a wide-based gait and some imbalance, she wears corrective lenses for hyperopia and is noted to have delayed tooth eruption and frequent ear infections. The patient babbles frequently and can speak 25-30 words. She is currently enrolled for physical, occupational, and developmental therapies through First Steps.

CMA identified a complex genomic rearrangement consisting of 28 breakpoints at 18q21.32q23 (56,820,380$77,916,234$ [hg19]) with 11 losses and 3 gains including a triplication for a total of 13.2 and $1.0 \mathrm{Mb}$ of genomic material affected, respectively. The size of the copy number alterations ranged from 0.042 to $5.1 \mathrm{Mb}$ with 12 small segments of normal copy number flanking these intervals (Fig. 2a). The specific intervals and genes within each of the copy number changes are summarized in Table 2 . The concurrent G-banded chromosome analysis identified an apparently terminal deletion of chromosome $18 \mathrm{q}$ (Fig. 2b). To ascertain the parent of origin, duo analysis was performed as the mother's sample could not be obtained. The biological father was determined to be negative for complex rearrangements on chromosome 18 (data not shown). SNP genotyping showed the presence of only maternal (non-paternal) alleles in the deleted regions of chromosome 18q21.32q23, suggesting that these complex rearrangements had arisen on the paternal chromosome (Fig. 2c).

\section{Discussion and Conclusion}

Here, we describe 2 new cases of complex intrachromosomal rearrangements with CMA profiles suggestive of germline chromoanagenesis events. While rare, chromoanagenesis in the constitutional setting has been reported in increasing frequency since the first description of the phenomenon in cancer, and is likely an underappreciated phenomenon in the germline [Piccione et al., 


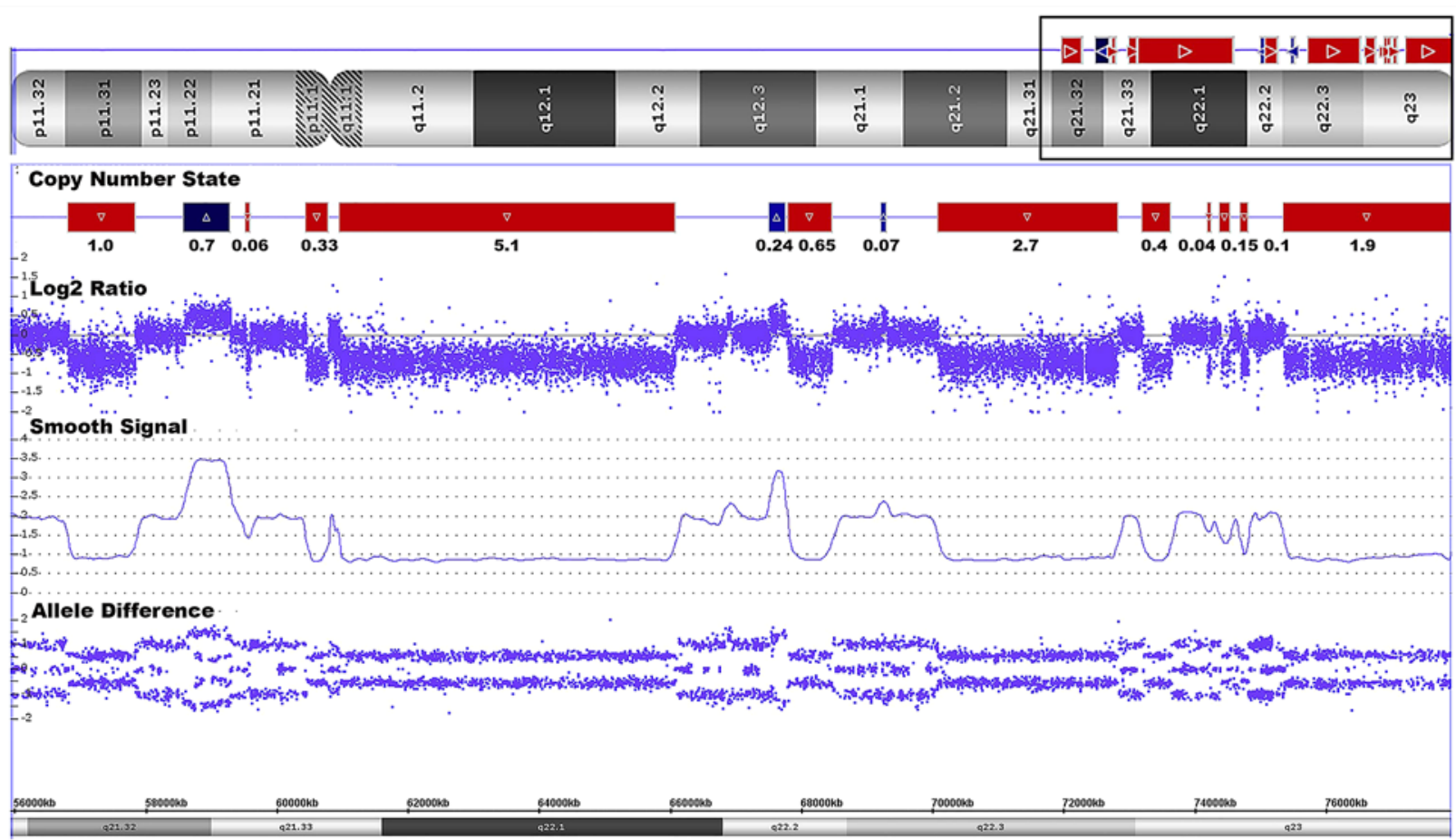

a

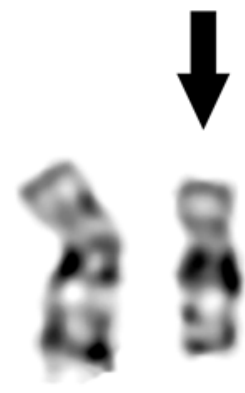

\begin{tabular}{cccc}
\hline $\begin{array}{c}\text { Chromosome 18 } \\
\text { Coordinate }\end{array}$ & SNP Marker & $\begin{array}{c}\text { Paternal } \\
\text { Genotype }\end{array}$ & $\begin{array}{c}\text { Proband's } \\
\text { Genotype }\end{array}$ \\
\hline 56827121 & S-3LTKN & BB & A \\
56827485 & S-4IVMR & BB & A \\
56832307 & S-4RNAV & AA & B \\
56877537 & S-4JHDY & AA & B \\
56878051 & S-4HJEX & AA & B \\
56878511 & S-3XEZA & AA & B \\
56878948 & S-3VFOB & AA & B \\
56913967 & S-3TPFK & BB & A \\
56920558 & S-3ZGRN & AA & B \\
56921310 & S-4MXNO & BB & A \\
68062692 & S-3BXRR & BB & A \\
68062834 & S-4PYMZ & BB & A \\
73481134 & S-3NXKH & AA & B \\
73481577 & S-4FZQD & AA & B \\
73486030 & S-4FIBG & AA & B \\
\hline
\end{tabular}

Fig. 2. Complex chromosomal rearrangement involving chromosome 18 in case 2 . a The boxed area on the chromosome 18 idiogram illustrates the region of detail shown below it. Chromosomal microarray data depict the complex chromosomal rearrangement at 18q21.32q23 (56,820,380-77,916,234 [hg19]). The alternating copy number losses and gains are indicated by the red and blue bars, respectively, with the size of individual CNVs listed below each in $\mathrm{Mb}$. Copy number analysis (Log2 ratio, smooth sig- nal) and genotype information (allele difference) are represented as individual tracks on the array plot. b G-banded partial karyotype showing the terminal deletion in one chromosome 18 (arrow). c Genotyping data for the duo (father and child) are represented for selected informative SNPs with specified chromosomal position and corresponding SNP marker. The paternal genotype is shown in red, and the derived genotype for the proband is depicted for deletions along the length of chromosome 18. 
Table 2. Specific intervals, size of copy number variants, and genes in the $18 \mathrm{q}$ region for case 2

\begin{tabular}{|c|c|c|c|}
\hline $18 \mathrm{q} 21.32\left(56820380 \_57837890\right) \times 1$ & 1.0 & SEC11C, GRP, RAX, CPLX4, LMAN1, CCBE1, PMAIP1 & VUS \\
\hline 18q21.33(59529458_59593278)×1 & 0.06 & RNF152 & VUS \\
\hline $18 \mathrm{q} 21.33\left(60448158 \_60780827\right) \times 1$ & 0.33 & PHLPP1 & VUS \\
\hline 18q21.33q22.1(60957693_66076119)×1 & 5.1 & $\begin{array}{l}\text { BCL2, KDSR, VPS4B, SERPINB5, SERPINB12, SERPINB13, SERPINB4, SERPINB3, } \\
\text { SERPINB11, SERPINB7, SERPINB2, SERPINB10, HMSD, SERPINB8, LINC00305, } \\
\text { LOC284294, LINC01538, CDH7, CDH19, MIR5011, DSEL, LOC643542 }\end{array}$ & $\begin{array}{l}\text { Likely } \\
\text { pathogenic }\end{array}$ \\
\hline $18 \mathrm{q} 22.2\left(67520222 \_67763031\right) \times 3$ & 0.24 & CD226, RTTN & VUS \\
\hline 18q22.2(67807620_68465752)×1 & 0.65 & RTTN, SOCS6, LOC101927481, LOC101060542, GTSCR1 & VUS \\
\hline 18q22.3(69225340_69297624)×3 & 0.07 & LINC01541 & \\
\hline $18 \mathrm{q} 22.3\left(70085574 \_72826738\right) \times 1$ & 2.7 & $\begin{array}{l}\text { CBLN2, NETO1, MIR548AV, LOC100505797, LOC400655, LOC100505817, FBXO15, } \\
\text { TIMM21, CYB5A, C18orf63, LOC101927606, FAM69C, CNDP2, CNDP1, } \\
\text { LINC00909, ZNF407 }\end{array}$ & VUS \\
\hline $18 \mathrm{q} 23\left(75,359,543 \_77,916,234\right) \times 1$ & 2.5 & $\begin{array}{l}\text { LINC01029, SALL3, ATP9B, NFATC1, LOC284241, CTDP1, KCNG2, PQLC1, } \\
\text { HSBP1L1, TXNL4A, RBFA, RBFADN, ADNP2, PARD6G-AS1, PARD6G }\end{array}$ & VUS \\
\hline
\end{tabular}

VUS, variant of uncertain significance.

2010; Kloosterman et al., 2011, 2012; Liu et al., 2011; Genesio et al., 2013, 2015; Gu et al., 2013; Kloosterman and Cuppen, 2013; Fontana et al., 2014; Nazaryan et al., 2014; Plaisancié et al., 2014; de Pagter et al., 2015; Gamba et al., 2015; Macera et al., 2015; Wang et al., 2015; Weckselblatt et al., 2015; Anderson et al., 2016; Bertelsen et al., 2016; Burnside et al., 2016; Del Rey et al., 2016; Masset et al., 2016; Fukami et al., 2017; Sabatini et al., 2018; Gudipati et al., 2019; Koltsova et al., 2019; Nazaryan-Petersen et al., 2019; Zepeda-Mendoza and Morton, 2019; Ader et al., 2020].

In each of our patients, conventional cytogenetics suggested simple deletions. This highlights the limited resolution of G-band analysis in these types of complex rearrangements, and suggests that some previously reported cases of simple deletions could in fact have unrecognized chromoanagenesis events. In these 2 cases, genomic microarray analysis further revealed the presence of numerous breaks with several copy number gains and losses in regions of chromosome $14 \mathrm{q}$ and $18 \mathrm{q}$, respectively. While the presence of duplications and triplications is a hallmark of chromoanasynthesis, the specific underlying DNA repair mechanism is currently undetermined in our cases, and thus we describe these rearrangements as being suggestive of the more encompassing descriptor germline chromoanagenesis. Undoubtedly, with the increasingly

Two Patients with Possible Germline Chromoanagenesis wide application of high-resolution molecular diagnostic methods such as next-generation sequencing in routine clinical practice, the detection and molecular characterization of such unbalanced catastrophic rearrangements in the germline will likely increase in the future.

Germline complex rearrangements can be stably transmitted from carrier parents or be the result of de novo events arising in either maternal or paternal germlines [Gruchy et al., 2010; Gu et al., 2013; de Pagter et al., 2015; Weckselblatt et al., 2015; Bertelsen et al., 2016; Del Rey et al., 2016; Collins et al., 2017; Grochowski et al., 2018; Sabatini et al., 2018; Gudipati et al., 2019]. While a preferential bias for paternal origin has been described in germline chromothripsis-mediated events, no parental preference has so far been described for chromoanasynthesis-derived rearrangements [Kloosterman et al., 2011, 2012; Liu et al., 2011; Weckselblatt and Rudd, 2015; Weckselblatt et al., 2015; Collins et al., 2017; Fukami et al., 2017; Zepeda-Mendoza and Morton, 2019]. In the current study, the complex rearrangements in both cases were determined to be paternal in origin. Indeed, further data need to be accrued to determine whether a parental bias exists for other forms of chromoanagenesis-compatible germline complex genomic rearrangements.

The patients in this study presented with variable phenotypes including developmental delay, hypotonia, and 
seizures. While the pathogenic nature of the rearrangement is evident in each case, a clear genotype-phenotype association was difficult to assess given the complexity of the rearrangements. Undoubtedly, the clinical consequence of any form of complex rearrangement is dependent on dosage effects of the genes located in the regions of copy number change, position effects related to the shuffling of regulatory elements, and the possible disruption of genes located at the breakpoints. Furthermore, the rare cases of phenotypically normal individuals harboring complex rearrangements highlight the additional influence of factors such as variable expressivity, incomplete penetrance, recessive inheritance, and the manifestation of sub-clinical phenotypes. The contributions of each of these effects in complex chromosomal rearrangements will be an important area for future investigation.

In conclusion, this report describes 2 patients with complex chromosome rearrangements having features characteristic of germline chromoanagenesis, most consistent with the subcategory of chromoanasynthesis. The rearranged chromosomes were of paternal origin in both patients, a result which may help elucidate whether preferential parental bias exists broadly in constitutional chromoanagenesis events or is specific to chromothripsis. Future studies such as breakpoint characterization by next-generation sequencing would provide a more detailed assessment of the structural rearrangements in these cases to further characterize the underlying repair mechanisms.

\section{Acknowledgements}

We would like to sincerely thank our patients and their families for participating and consenting to this study. We would also like to thank the referring physicians Dr. Robert Ward and Dr. Molly $\mathrm{McPheron}$ for their invaluable contributions.

\section{Statement of Ethics}

Written informed consent was obtained from each patient's guardian/parents to participate in this research through our institutional research ethics board protocol (IRB \#1011003014).

\section{Conflict of Interest Statement}

The authors have no conflicts of interest to declare.

\section{Funding Sources}

The authors have no funding sources to report.

\section{Author Contributions}

P.A. and A.M.B. drafted the manuscript. P.A.M. and P.A. generated and analyzed the data. A.M.B. and J.C.H. contributed to the review and interpretation of the data. P.A., J.C.H., G.H.V., and A.M.B. contributed to the critical review of the manuscript. All authors read and approved the final manuscript.

\section{References}

Ader F, Heide S, Marzin P, Afenjar A, Diguet F, Chantot Bastaraud S, et al. A 14q distal chromoanagenesis elucidated by whole genome sequencing. Eur J Med Genet. 2020;63(4): 103776.

Anderson SE, Kamath A, Pilz DT, Morgan SM. A rare example of germ-line chromothripsis resulting in large genomic imbalance. Clin Dysmorphol. 2016;25(2):58-62.

Baca SC, Prandi D, Lawrence MS, Mosquera JM, Romanel A, Drier Y, et al. Punctuated evolution of prostate cancer genomes. Cell. 2013; 153(3):666-77.

Bertelsen B, Nazaryan-Petersen L, Sun W, Mehrjouy MM, Xie G, Chen W, et al. A germline chromothripsis event stably segregating in 11 individuals through three generations. Genet Med. 2016;18(5):494-500.

Bone K, MacPherson MJ, Chernos J, Lauzon J. Failure of NIPT to detect constitutional chromoanasynthesis involving chromosome 21 in a case of fetal hydrops-A case report. Clin Case Rep. 2019;7(11):2165-8.
Burnside RD, Harris A, Speyer D, Burgin WS, Rose DZ, Sanchez-Valle A. Constitutional Chromoanagenesis of Distal 13q in a Young Adult with Recurrent Strokes. Cytogenet Genome Res. 2016;150(1):46-51.

Collins RL, Brand H, Redin CE, Hanscom C, Antolik C, Stone MR, et al. Defining the diverse spectrum of inversions, complex structural variation, and chromothripsis in the morbid human genome. Genome Biol. 2017;18(1):36. de Pagter MS, van Roosmalen MJ, Baas AF, Renkens I, Duran KJ, van Binsbergen E, et al. Chromothripsis in healthy individuals affects multiple protein-coding genes and can result in severe congenital abnormalities in offspring. Am J Hum Genet. 2015;96(4):651-6.

Del Rey J, Santos M, González-Meneses A, Milà M, Fuster C. Heterogeneity of a Constitutional Complex Chromosomal Rearrangement in 2q. Cytogenet Genome Res. 2016;148(2-3): $156-64$.

Fontana P, Genesio R, Casertano A, Cappuccio G, Mormile A, Nitsch L, et al. Loeys-Dietz syn- drome type 4 , caused by chromothripsis, involving the TGFB2 gene. Gene. 2014;538(1): 69-73.

Fukami M, Shima H, Suzuki E, Ogata T, Matsubara K, Kamimaki T. Catastrophic cellular events leading to complex chromosomal rearrangements in the germline. Clin Genet. 2017; 91(5):653-60.

Gamba BF, Richieri-Costa A, Costa S, Rosenberg C, Ribeiro-Bicudo LA. Chromothripsis with at least 12 breaks at $1 \mathrm{p} 36.33-\mathrm{p} 35.3$ in a boy with multiple congenital anomalies. Mol Genet Genomics. 2015;290(6):2213-6.

Genesio R, Ronga V, Castelluccio P, Fioretti G, Mormile A, Leone G, et al. Pure 16q21q22.1 deletion in a complex rearrangement possibly caused by a chromothripsis event. Mol Cytogenet. 2013;6(1):29.

Genesio R, Fontana P, Mormile A, Casertano A, Falco M, Conti A, et al. Constitutional chromothripsis involving the critical region of 9q21.13 microdeletion syndrome. Mol Cytogenet. 2015;8:96. 
Grochowski CM, Gu S, Yuan B, Tcw J, Brennand KJ, Sebat J, et al. Marker chromosome genomic structure and temporal origin implicate a chromoanasynthesis event in a family with pleiotropic psychiatric phenotypes. Hum Mutat. 2018;39(7):939-46.

Gruchy N, Barreau M, Kessler K, Gourdier D, Leporrier N. A paternally transmitted complex chromosomal rearrangement (CCR) involving chromosomes 2,6 , and 18 includes eight breakpoints and five insertional translocations (ITs) through three generations. Am J Med Genet A. 2010;152A(1):185-90.

Gu H, Jiang JH, Li JY, Zhang YN, Dong XS, Huang YY, et al. A familial Cri-du-Chat $/ 5 p$ deletion syndrome resulted from rare maternal complex chromosomal rearrangements (CCRs) and/or possible chromosome $5 \mathrm{p}$ chromothripsis. PLoS One. 2013;8(10): e76985.

Gudipati MA, Waters E, Greene C, Goel N, Hoppman NL, Pitel BA, et al. Stable transmission of complex chromosomal rearrangements involving chromosome $1 \mathrm{q}$ derived from constitutional chromoanagenesis. Mol Cytogenet. 2019;12:43.

Holland AJ, Cleveland DW. Chromoanagenesis and cancer: mechanisms and consequences of localized, complex chromosomal rearrangements. Nat Med. 2012;18(11):1630-8.

Kloosterman WP, Cuppen E. Chromothripsis in congenital disorders and cancer: similarities and differences. Curr Opin Cell Biol. 2013; 25(3):341-8.

Kloosterman WP, Guryev V, van Roosmalen M, Duran KJ, de Bruijn E, Bakker SC, et al. Chromothripsis as a mechanism driving complex de novo structural rearrangements in the germline. Hum Mol Genet. 2011;20(10): 1916-24.

Kloosterman WP, Tavakoli-Yaraki M, van Roosmalen MJ, van Binsbergen E, Renkens I, Du- ran $\mathrm{K}$, et al. Constitutional chromothripsis rearrangements involve clustered doublestranded DNA breaks and nonhomologous repair mechanisms. Cell Rep. 2012;1(6):64855.

Koltsova AS, Pendina AA, Efimova OA, Chiryaeva OG, Kuznetzova TV, Baranov VS. On the Complexity of Mechanisms and Consequences of Chromothripsis: An Update. Front Genet. 2019;10:393.

Liu P, Erez A, Nagamani SC, Dhar SU, Kołodziejska KE, Dharmadhikari AV, et al. Chromosome catastrophes involve replication mechanisms generating complex genomic rearrangements. Cell. 2011;146(6): 889-903.

Ly P, Cleveland DW. Rebuilding Chromosomes After Catastrophe: Emerging Mechanisms of Chromothripsis. Trends Cell Biol. 2017; 27(12):917-30.

Macera MJ, Sobrino A, Levy B, Jobanputra V, Aggarwal V, Mills A, et al. Prenatal diagnosis of chromothripsis, with nine breaks characterized by karyotyping, FISH, microarray and whole-genome sequencing. Prenat Diagn. 2015;35(3):299-301.

Masset H, Hestand MS, Van Esch H, Kleinfinger P, Plaisancié J, Afenjar A, et al. A Distinct Class of Chromoanagenesis Events Characterized by Focal Copy Number Gains. Hum Mutat. 2016;37(7):661-8.

Nazaryan L, Stefanou EG, Hansen C, Kosyakova N, Bak M, Sharkey FH, et al. The strength of combined cytogenetic and mate-pair sequencing techniques illustrated by a germline chromothripsis rearrangement involving FOXP2. Eur J Hum Genet. 2014;22(3):33843.

Nazaryan-Petersen L, Oliveira IR, Mehrjouy MM, Mendez JMM, Bak M, Bugge M, et al. Multigenic truncation of the semaphorin-plexin pathway by a germline chromothriptic rear- rangement associated with Moebius syndrome. Hum Mutat. 2019;40(8):1057-62.

Pellestor F. Chromoanagenesis: cataclysms behind complex chromosomal rearrangements. Mol Cytogenet. 2019;12:6.

Piccione M, Antona V, Scavone V, Malacarne M, Pierluigi M, Grasso M, et al. Array CGH defined interstitial deletion on chromosome 14: a new case. Eur J Pediatr. 2010;169(7):84551.

Plaisancié J, Kleinfinger P, Cances C, Bazin A, Julia S, Trost D, et al. Constitutional chromoanasynthesis: description of a rare chromosomal event in a patient. Eur J Med Genet. 2014;57(10):567-70.

Sabatini PJB, Ejaz R, Stavropoulos DJ, MendozaLondono R, Joseph-George AM. Stable transmission of an unbalanced chromosome $21 \mathrm{de}$ rived from chromoanasynthesis in a patient with a SYNGAP1 likely pathogenic variant. Mol Cytogenet. 2018;11:50.

Stephens PJ, Greenman CD, Fu B, Yang F, Bignell GR, Mudie LJ, et al. Massive genomic rearrangement acquired in a single catastrophic event during cancer development. Cell. 2011; 144(1):27-40.

Wang JC, Fisker T, Sahoo T. Constitutional chromothripsis involving chromosome 19 in a child with subtle dysmorphic features. Am J Med Genet A. 2015;167A(4):910-3.

Weckselblatt B, Rudd MK. Human Structural Variation: Mechanisms of Chromosome Rearrangements. Trends Genet. 2015;31(10): 587-99.

Weckselblatt B, Hermetz KE, Rudd MK. Unbalanced translocations arise from diverse mutational mechanisms including chromothripsis. Genome Res. 2015;25(7):937-47.

Zepeda-Mendoza CJ, Morton CC. The Iceberg under Water: Unexplored Complexity of Chromoanagenesis in Congenital Disorders. Am J Hum Genet. 2019;104(4):565-77.
Two Patients with Possible Germline Chromoanagenesis
Cytogenet Genome Res 2020;160:671-679 